\title{
e-Phaïstos
}

e-Phaïstos

Revue d'histoire des techniques / Journal of the history

of technology

VII-1 | 2019

Le travail humain

\section{Les travaux sur la fatigue humaine à l'épreuve du terrain.}

Le cas du système Gennaper dans les mines de Pertusola (1930-1940)

Testing Human Fatigue's Studies on the Field: the Case of the Gennaper System in the Peñarroya Mines (1930-1940)

\section{Francesca SANNA}

\section{OpenEdition}

\section{Journals}

Édition électronique

URL : http://journals.openedition.org/ephaistos/4079

DOI : 10.4000/ephaistos.4079

ISSN : 2552-0741

Éditeur

IHMC - Institut d'histoire moderne et contemporaine (UMR 8066)

\section{Référence électronique}

Francesca SANNA, «Les travaux sur la fatique humaine à l'épreuve du terrain. », e-Phaïstos [En ligne], VII-1 | 2019, mis en ligne le 06 avril 2019, consulté le 01 mai 2019. URL : http://

journals.openedition.org/ephaistos/4079 ; DOI : 10.4000/ephaistos.4079

Ce document a été généré automatiquement le 1 mai 2019.

Tous droits réservés 


\title{
Les travaux sur la fatigue humaine à l'épreuve du terrain.
}

\author{
Le cas du système Gennaper dans les mines de Pertusola (1930-1940) \\ Testing Human Fatigue's Studies on the Field: the Case of the Gennaper System \\ in the Peñarroya Mines (1930-1940)
}

Francesca SANNA

\section{Introduction}

Dans le contexte de la rationalisation d'entre-deux-guerres, on voit la naissance de plusieurs modèles d'évaluation qui proposent d'optimiser la performance du corps au travail par la gestion de sa dépense énergétique. La conception de ces techniques croise plusieurs types de savoirs, en premier lieu les études sur la fatigue humaine des XVIII et $\mathrm{XIX}^{\mathrm{e}}$ siècles et les applications de l'organisation rationnelle. La mise à l'épreuve du terrain donne ensuite lieu à des procédés d'adaptation et modification, qui mêlent l'empirisme de la pratique à la maîtrise de la technique. L'objectif étant la minimisation des coûts de revient, l'intervention sur le travail humain doit résulter d'une transformation du savoir en outil, pour mesurer, discipliner et optimiser la performance en fonction de son coût. La recherche de ces solutions adaptatives engendre parfois des dynamiques de circulation des savoirs, dans lesquelles le rôle intermédiaire des ingénieurs est essentiel. Cette figure professionnelle semble en fait condenser connaissance théorique et expérience pratique pour une finalité évaluative du travail. À partir d'un cas d'étude concernant le monde de la mine, cette contribution observe la mise au profit du savoir sur le travail humain au sein d'un projet de rationalisation. On analysera ainsi l'histoire d'un conflit ouvrier opposant, en Italie, le syndicat fasciste à une compagnie minière italienne, contrôlée par un groupe multinational et dirigée par un ingénieur français. À la suite de ce conflit, qui touche le projet de rationalisation de la dite compagnie, une dynamique de circulation transnationale des savoirs s'active et conduit à l'élaboration d'une nouvelle technique d'évaluation du travail. On verra donc quels sont les savoirs mobilisés et la manière dont ils sont mobilisés. On observera aussi les outils et les procédés du travail créatif des 
ingénieurs et enfin on en examinera le résultat à la lumière des sources disponibles. Ces sources comprennent notamment une partie des archives de la compagnie minière, conservées à l'Archivio Storico Minerario della Sardegna (Iglesias) et les articles, au sujet de la nouvelle technique, publiés par l'ingénieur français Paul Audibert dans la Revue de l'Industrie Minérale.

\section{La rationalisation de la fatigue : un savoir conflictuel}

2 Les études sur la fatigue humaine viennent du terrain. C'est en effet en observant les travaux de construction de l'aqueduc de Maintenon que Vauban chercha les conditions de la meilleure performance en combinant différemment la vitesse et le poids dans les opérations de terrassement. Il en fut de même pour Coulomb, qui réfléchit au poids optimal de la charge des manutentionnaires dans le Mémoire sur la force des hommes à partir de l'expérience du pic de Tenerife. L'idée d'attaquer le problème de l'effort par l'étude de ses symptômes apparents est donc à l'origine du concept de la fatigue au travail, qui se sublime en problème scientifique dans différentes disciplines comme la physique, la physiologie ou la médecine. Avec les études de laboratoire, la modélisation de différents « symptômes » de la fatigue se fait par le biais de l'expérience contrôlée. On construit alors des outils et des instruments de mesure, on se plonge dans les applications de calorimétrie et thermodynamique, on étudie les effets des conditions externes, de l'alimentation, de l'hygiène de vie ${ }^{1}$. Cependant, malgré tous ces efforts, la fatigue reste un objet scientifique insaisissable.

3 À partir de la fin du XIX siècle, l'influence de la fatigue sur les performances humaines dans l'industrie commence à susciter l'intérêt de ceux qui s'interrogent sur la mise en place de la rationalisation du travail. Dans ce contexte, on ne peut pas ignorer le poids des études de Taylor qui, d'un certain point de vue, hégémonisent le discours concernant rationalisation et organisation du travail. Toutefois les ouvrages de Taylor délivrent une image étroitement liée à une conception machiniste de l'homme au travail ${ }^{2}$. Malgré quelques passages génériques sur la question de l'épuisement, le silence de Taylor au sujet de la fatigue devient un problème lors que les "organisateurs de la production " ${ }^{3}$ (consultants ou ingénieurs) commencent à imaginer des outils complexes d'évaluation du travail humain. Dans cette perspective, la reprise des études du travail au sein d'une réflexion liée au management industriel caractérise le virage post-taylorien de ce qui a été appelé "efficiency craze $»^{4}$. Cette course à l'optimisation exige en effet une adaptation des connaissances scientifiques à des objectifs économiques. En d'autres termes, on vise l'optimisation de la performance énergétique du corps humain face aux contraintes matérielles du lieu de travail ${ }^{5}$. La transformation des lieux du travail et du régime disciplinaire qui encadre les ouvriers ne se fait pourtant pas sans difficultés. Le conflit généré par l'application de méthodes de rationalisation cible surtout l'accélération des rythmes de travail et sur les effets de surmenage et d'épuisement qui en résultent. On voudrait ici proposer le cas d'étude des mines de la Compagnie Pertusola, qui permet de discuter la question de l'analyse technique de la fatigue comme un élément caractérisant de ce genre de conflit.

4 L'irruption du problème de la fatigue dans les mines italiennes de la Compagnie Pertusola suit le début d'un projet de rationalisation par l'application du système Bedaux ${ }^{6}$. Salué avec enthousiasme par les ingénieurs, ce nouveau système suscite la protestation ouvrière et syndicale, qui cible surtout les célèbres "coefficients d'effort» du système. 
Ceux-ci sont supposés représenter fidèlement la dépense énergétique (l'effort) qui est nécessaire pour accomplir une tâche sans provoquer l'épuisement à la fin de la journée de travail. Ces coefficients représentent également la base de la rémunération Bedaux, dans une logique qui voudrait payer le travailleur proportionnellement à l'énergie dépensée et non pas au travail accompli. Dans le discours de Charles E. Bedaux, l'optimisation de la gestion énergétique doit permettre une amélioration de la performance et, en conséquence, la possibilité d'intensifier la productivité par l'augmentation de l'allure.

5 L'évaluation (la mesure) est donc à la fois un outil d'étude et une forme de contrainte du travail. De ce point de vue, les " coefficients d'effort », considérés comme arbitraires par les syndicats, sont également dénoncés comme une cause de surexploitation des ouvriers. Aussi, dans les mines de Pertusola, l'opposition au système Bedaux survient entre 1929 et $1931^{7}$. Les ouvriers n'utilisent jamais l'arme de la grève et on constate plutôt le recours aux pratiques d'insubordination, surtout dans une forme de mépris verbal envers les chronométreurs et par de plus rares actions de freinage ${ }^{8}$. Les raisons de ce comportement, qu'on observe aussi parmi les travailleurs d'autres compagnies de la région, sont probablement à rechercher dans le contexte de souffrance économique provoqué par la crise minière des années 1920 et dans la répression fasciste des mouvements syndicaux qui empêche le déploiement d'une opposition plus organisée. Il faut également considérer le profil socio-culturel du territoire, profondément ancré à une dimension rurale, très peu industrialisé, ce qui retarde la naissance d'une véritable communauté ouvrière et des mouvements syndicaux ${ }^{9}$. Les formes de contestation sont donc ponctuelles et désorganisées.

Cette tension attire en tous cas l'attention du syndicat fasciste local qui met en lumière certaines irrégularités dans la mise en place du nouveau régime salarial. L'attitude inflexible des compagnies minières face à ces revendications provoque ensuite l'intervention des autorités confédérales ${ }^{10}$. Le conflit se déplace au niveau national: la Confédération des syndicats fascistes de l'Industrie engage l'Association Nationale des Industriels qui, à son tour, renvoie la question à l'Union des Industriels sardes, qui opère la médiation avec les compagnies minières locales. Les échanges reprennent les accusations portées au système Bedaux : provoquer la baisse des salaires, le chômage et créer une situation de souffrance psycho-physique au travail. La négociation est difficile car, d'un côté, les compagnies ne cèdent rien à leur position et, de l'autre, le Président de la Confédération, Tullio Cianetti, est ouvertement hostile au système Bedaux (malgré l'ambivalence générale des autorités fascistes au sujet de l'OST) ${ }^{11}$. Le conflit prend donc une tournure plus politique qui devient très évidente lors de la visite de T. Cianetti en Sardaigne, en novembre 1934. Du côté ouvrier, l'opposition semble par ailleurs désactivée : les épisodes d'insubordination diminuent, les mineurs ne sont que rarement en grève et ils montrent, en général, une attitude d'« acceptation » envers le nouveau régime de travail ${ }^{12}$. Si, comme on l'a évoqué, la combattivité des mineurs sardes est faible, il faut aussi considérer que le conflit se prolonge dans le temps, loin du territoire local, et porte sur un sujet complexe, le problème des coefficients, ce qui défavorise une action sur le terrain.

7 Dans ce contexte, les ingénieurs de Pertusola, coordonnés par Paul Audibert, Inspecteur de la compagnie à Gênes, entament au printemps 1932 une étude de vérification des coefficients Bedaux, qui représentent le véritable objet de la contestation syndicale. Cette étude correspond à la première étape d'une recherche sur la fatigue humaine et de l'élaboration de la méthode Gennaper, qui fait l'objet de cet article. Loin de représenter 
un rapprochement aux positions ouvrières ou une "préoccupation d'industriels humanitaires ${ }^{13}$, comme le dit Alexandre Lhéraud, directeur de Pertusola, cette étude semble plutôt avoir les traits d'une contre-mesure préventive. En effet, au printemps 1932 les mineurs de Gavorrano, en Toscane, obtiennent, à la suite d'une grève assez violente, l'abolition du système Bedaux par la Compagnie Montecatini ${ }^{14}$. L'événement est traité dans la correspondance entre A. Lhéraud et l'Union des Industriels sardes, qui avait envoyé un questionnaire à la compagnie Pertusola au sujet du système Bedaux. Dans ses réponses, A. Lhéraud évoque les études Gennaper. Les sources semblent suggérer que ces études sont entamées pour prévenir des situations comme celle de Montecatini. Cependant, à la fin de l'année 1934, les accords séparés de FIAT et Montecatini au sujet du système Bedaux donnent une impulsion à la négociation au niveau national ${ }^{15}$. Les compagnies minières sardes ne peuvent qu'accepter la résolution de mars 1935 (Patti di Marzo) qui abolit formellement le système Bedaux. La Confédération syndicale clame la victoire, mais l'accord ne change que partiellement les conditions du travail des mineurs de Pertusola, soumis dès la fin de l'année 1933 à l'application d'une nouvelle technique, le Gennaper ${ }^{16}$.

8 Les techniques de rationalisation font donc l'objet privilégié, comme souvent au $\mathrm{XX}^{\mathrm{e}}$ siècle, d'un conflit qui oppose les ouvriers au patronat et qui implique aussi les institutions. Plus spécifiquement, le replacement politique du conflit transforme la technique en objet de négociation, ce qui met en cause les formes et les limites de la discipline du travail. Dans le cas de Pertusola, le conflit institutionnel se maintient loin du terrain local, grâce à l'action d'A. Lhéraud qui gère les relations par une communication purement épistolaire. Le risque d'un retour du conflit sur le carreau des mines est donc limité, d'autant plus que les ouvriers abandonnent progressivement la contestation. Les ingénieurs de Pertusola peuvent donc développer leurs études dans un milieu assez favorable, quoi que parfois instable. Le projet de recherche apparait enfin comme une solution originale au conflit, qui permet d'obtenir un apaisement des tensions et de garder en même temps l'intégralité des fonctions disciplinaires de l'OST.

Dans la prochaine partie, on discutera donc comment, grâce à une circulation transnationale des savoirs concernant la fatigue humaine au travail, les ingénieurs de Pertusola peuvent élaborer et mettre en place leur propre solution d'OST.

\section{Étudier la fatigue humaine au travail : un objet de circulation des savoirs}

10 Au printemps 1932, l'équipe de recherche Pertusola, composée de quatre ingénieurs sous la direction de $\mathrm{P}$. Audibert, se propose de donner une réponse à la demande de vérification des coefficients Bedaux. Les coefficients doivent être discutés selon deux pistes d'analyse: d'une part, l'appréciation de la vitesse du travail, et d'autre part, l'évaluation de la puissance du travail. Mais les premiers résultats de l'enquête suggèrent la possibilité d'aller au-delà d'une simple vérification et de développer une méthode d'évaluation totalement nouvelle, le Gennaper. Cette innovation va faire l'objet de trois publications dans la Revue de l'Industrie Minérale entre 1933 et $1937^{17}$. La comparaison de ces articles aux fonds d'archives met en évidence le croisement de plusieurs approches quant au problème de la fatigue. Comme nous allons le voir, les ingénieurs rassemblent des références scientifiques qu'ils utilisent au fur et à mesure pour aider à la compréhension du problème de la fatigue. Ces théories - alors transformées en véritables 
outils d'analyse - posent les bases du traitement des données expérimentales. L'étude Gennaper (qui relève d'une scientificité parfois empirique) nous permet donc d'observer de plus près la vision de la fatigue partagée par les ingénieurs, et la façon dont ils traitent le problème du travail à la mine.

Dans la première publication de 1933, P. Audibert présente un encadrement théorique du problème de la fatigue qui reflète en partie la littérature de référence de l'équipe Pertusola. L'esquisse concerne d'une part la science de la rationalisation du travail et, de l'autre, la littérature sur la fatigue ${ }^{18}$. Pour ce qui concerne la première partie, la «science de l'organisation rationnelle » est associée à deux figures principales, Taylor et Bedaux, mais l'analyse porte plus spécifiquement sur la question des "coefficients d'effort». Pour l'auteur, promoteur du système chez Pertusola, Bedaux apporte "plus de science, de précision et de justice $»^{19}$, mais ne donne pas de solution au problème de l'optimisation du geste technique. L'étude de vérification pourrait donc résoudre non seulement le conflit ouvrier, mais aussi les problèmes techniques du système Bedaux. Dans la deuxième partie de l'article, on cite les "systèmes sans chronométrage et études théoriques ", qui comptent des auteurs (issus de disciplines diverses) tels que: Atwater, Benedict et Hirn (thermodynamique); Mosso, Maggiora, Treves, Ioteyko et Chauveau (physiologie); Meyerhoff et Hill (biologie et chimie) ; Zuntz, Schumburg et Marey (pour leurs travaux sur la marche); et enfin Jules Amar et Armand Imbert (pour leur étude des «diverses modalités du travail professionnel »). Cette littérature - qui reste mobilisée à l'état d'évocation, à travers des citations - compose une sorte de summa : un univers scientifique de référence à la lumière duquel on cherche une solution au problème du travail humain. Cependant, la conclusion qu'en tire l'auteur est tranchante :

\footnotetext{
«Tout donne l'impression d'un chantier trop vaste pour les équipes de maçons au travail avec une liaison insuffisante entre les diverses équipes. Voilà pourquoi les industriels n'ont pas encore été attirés par les méthodes énergétiques d'évaluation du travail $»^{20}$.
}

P. Audibert constate ainsi un certain détachement de la littérature par rapport aux exigences réelles de la production, qui se reflète dans une évocation par énumération comme miroir de son inefficacité. Pourtant cette littérature forme une contextualisation nécessaire pour définir le problème de l'évaluation du travail et ensuite présenter les études Gennaper comme l'heureuse rencontre - nous dirions presque "l'aboutissement " - de ces différentes traditions. C'est donc dans cette perspective que les ingénieurs commencent à jeter les bases de la nouvelle méthode. Enfin, P. Audibert discute plus longuement de quatre catégories d'études : les études mathématiques et physiques (Euler, La Hire, Bernoulli, Coulomb) ; les études thermodynamiques (Atwater, Benedict, Hirn); les travaux de Chauveau; et enfin les "études sur la fatigue par ses signes" (Lavoisier, Mosso, Henry et Ioteyko, Hele-Shaw) ${ }^{21}$. Ces pistes constituent la véritable bibliographie de référence des ingénieurs effectivement mobilisée pour procéder à l'analyse expérimentale.

Les ouvrages véritablement exploités sont résumés, traduits en italien et classés par sujet dans les rapports techniques qu'on retrouve dans les fonds d'archive. Ainsi les travaux de Mosso et Trèves sont rangés dans la catégorie «Variation de l'amplitude et régularité du mouvement musculaire avec enregistrement mécanique et électrique ", tandis qu'Amar est associé à la mesure de la «Diminution de la force des muscles au dynamomètre ». Il en résulte une lecture sélective qui a pour but la recherche des lois applicables aux cas concrets. Par 
exemple, les deux résumés sur Angelo Mosso relatent de manière générale sa conception de la fatigue, mais les images des outils de mesure sont reproduites soigneusement. Les connaissances acquises à travers cette revue de littérature sont enfin rassemblées dans un mémoire intitulé « Résumé Récapitulatif» datant d'avril 193322. Ce mémoire compare les ouvrages de cinq auteurs (Mosso, Broca, Amar, Herlitzka et Ioteyko) sur sept thèmes : la fatigue musculaire, la fatigue intellectuelle, le repos, les phénomènes compensatoires, la production au travail et le rendement, la loi de la fatigue. Par rapport à l'article de P. Audibert, le mémoire fait référence à moins d'ouvrages, en sélectionnant les titres les plus récents ou les summae d'un domaine. Il s'agit enfin d'un vademecum qui expose les controverses principales au sujet de la fatigue, une synthèse dont on extrait une interprétation assez fonctionnelle.

On voit donc que les ingénieurs réutilisent surtout les ouvrages de Coulomb, Amar et Mosso. En ce qui concerne Mosso, les ingénieurs montrent une approche comparative assez élémentaire: ils observent les similitudes graphiques entre leurs courbes et les ergogrammes. Il nous semble que cela est dû au fait que, n'ayant pas pour but d'expliquer les causes physiologiques de la fatigue, les ingénieurs considèrent que les ergogrammes représentent un modèle - une sorte de fac-similé - pour détecter la présence de fatigue musculaire à travers un procédé analogique ${ }^{23}$. Cependant, ces courbes avaient déjà été largement critiquées par les physiologistes, notamment Trèves. P. Audibert connaît les travaux de ce dernier, mais l'usage comparatif des ergogrammes n'est abandonné qu'à fin de développer de nouvelles "courbes de fatigue ». Pour cela, les ingénieurs reviennent aux études de la physique mécanique. Si, du côté physiologique, cela peut paraître une régression, de l'autre côté, ce choix semble assez cohérent avec les propos et les moyens techniques à leur disposition. Plus spécifiquement, l'équipe choisit de parcourir la voie de la puissance pour évaluer l'effort, sans passer par l'appréciation de la vitesse (et donc par les contestés chronométrages). Cette intuition est à la fois le résultat d'un calcul de convenance et d'une réflexion autour de l'énergie humaine au travail. Forts de l'observation du geste technique et du corps humain dans la mine, les ingénieurs reconnaissent l'importance de la question énergétique pour l'efficience humaine dans l'activité extractive. Or, dans la définition physique du travail, la seule alternative à l'appréciation de la vitesse étant l'évaluation de la puissance, les ingénieurs s'appuient sur les lois d'Euler et Coulomb pour jeter une première base à leur méthode alternative.

Quelle est donc la conception de la fatigue qui inspire ces techniciens? D'une part, elle repose sur deux auteurs fondamentaux (Amar et Mosso), mais d'autre part, le modèle expérimental s'appuie sur l'application des lois physiques de Coulomb et Euler. En considérant le geste comme action technique productive, la fatigue est donc indiquée comme l'effet d'une dysfonction, à la fois du geste (mauvaise exécution, mauvaise posture, vitesse exagérée) ou de l'organisation du processus productif (conditions structurelles ou contingentes caractérisant l'environnement du travail) ${ }^{24}$. La fatigue interviendrait alors en conséquence de cette dysfonction, en tant que symptôme d'un déséquilibre entre les possibilités psycho-physiques du corps ouvrier et la demande énergétique exigée par le travail. Comment alors mesurer l'ampleur de cette dysfonction et $\mathrm{y}$ porter solution? 


\section{Transformer les savoirs en outils : les connaissances à l'épreuve du terrain}

17 Le traitement que les ingénieurs font subir à la loi de Coulomb montre comment l'équipe retravaille et met en perspective les connaissances acquises dans la littérature. Rappelons tout d'abord qu'ils désignent comme "loi de Coulomb » l'énoncé qui affirme qu'un homme ne peut pas donner quotidiennement plus de $4 / 9^{\text {e }}$ de son effort maximal possible, et que dans le cas de l'élévation d'une charge, la vitesse ne doit pas dépasser $1 / 3$ de la vitesse à vide. En réalité, cet énoncé appartient non pas à Coulomb, mais à Euler, puisque la « loi de Coulomb » affirme que le poids optimum de la charge (dans le travail de manutention) ne peut pas dépasser les $4 / 5^{e}$ du poids de l'homme. Ce malentendu vient du fait que l'énoncé de Coulomb est appris par la lecture d'un ouvrage de René Sand, qui le lui attribue ${ }^{25}$; c'est seulement après la lecture de l'ouvrage de Jules Amar qu'ils s'aperçoivent de l'erreur. Pour Jules Amar, Coulomb est «le plus grand de tous", le précurseur idéal de Taylor et Gilbreth car " il sut allier la théorie à l'expérimentation $»^{26}$. Cette proximité est peut-être une des raisons sous-jacentes au choix de revenir aux études de Coulomb plutôt que continuer sur le chemin de la psycho-physiologie. La discipline de la physique apparait, en plus, plus proche de la formation des ingénieurs par rapport aux savoirs biologiques et médicaux qui soutiennent la psycho-physiologie.

Or, l'hypothèse de « loi de Coulomb-Euler » fait l'objet d'un véritable test de terrain. Cette confrontation donne lieu à un débat au sein de l'équipe à la suite de l'application peu orthodoxe opérée par un des ingénieurs de l'équipe, le belge Maurice Bremer. Si P. Audibert désapprouve d'abord ce choix - et non sans une certaine ironie : «Il s'agit là d'une loi de Bremer et non plus de la loi de Coulomb", commente-t-il -, Bremer se justifie à l'aide d'observations expérimentales ${ }^{27}$. L'argumentation semble finalement convaincre $P$. Audibert, si bien que l'on retrouve cette résolution dans l'article de la Revue de l'Industrie Minérale. Les expériences Bremer donnent comme résultat des "courbes effort-vitesse caractéristiques » qui sembleraient être en rapport constant avec les courbes d'Euler. Pour justifier ces résultats, P. Audibert rappelle les études de Schultze et Langsdorf, en reprenant une fois de plus l'ouvrage de J. Amar. Cependant, les travaux de ces mathématiciens ne sont évoqués que dans l'article, car les sources nous montrent que la démonstration de l'équipe se fait sur une base purement expérimentale, notamment par une série d'études de temps au chronomètre ${ }^{28}$.

19 À la fin de l'année 1934, l'équipe Pertusola met un terme aux études. Le résultat final est une modélisation de l'effort humain en cinq courbes, à partir desquelles on tire des coefficients de puissance pour les tâches de poussée, traction et marche avec fardeaux. Avec ces coefficients, les ingénieurs estiment le temps standard exigible pour exécuter une tâche, en mesurant des grandeurs complémentaires comme le poids du fardeau. On peut donc affirmer, contrairement à ce qui a été auparavant suggéré, que le Gennaper n'est pas une méthode de rémunération ni une simple étude de vérification des coefficients Bedaux ${ }^{29}$. Il serait plutôt le résultat d'un effort de compréhension du travail humain, finalisé avec son optimisation industrielle. Il demeure une expérience d'exception et il diffère du système Bedaux non seulement pour ses caractères techniques, mais aussi pour l'absence d'un coté rémunératoire et, évidemment, d'un marchandising. L'histoire du Gennaper est donc plus proche d'une histoire des sciences et des techniques, plutôt que de la consultance managériale. Son application, qu'on 
abordera dans la prochaine partie de cet article, représente en tous cas pour Pertusola et ses ingénieurs le déploiement d'un outil de gestion et de contrôle de la main d'œuvre.

\section{La mise en place de la technique : est-il possible de rationaliser la fatigue?}

La comparaison des sources d'archives et imprimées montre que le système Gennaper s'appuie sur la réception et l'adaptation de savoirs provenant d'une circulation médiatisée par les ingénieurs. La nature de ces études est pourtant éminemment expérimentale. Cette manière de procéder repose sur une forma mentis spécifique aux ingénieurs : placés au carrefour de la science et de la technique, ces professionnels sont formés pour être scientifiquement pragmatiques. En d'autres termes, ils s'emploient à trouver des solutions adaptatives aux besoins contingents, en faisant appel à des solides connaissances théoriques et à une approche appliquée reposant sur différents procédés inventifs, dont notamment l'analogie ${ }^{30}$. Comme l'évoque Philippe Jamet dans une métaphore suggestive, l'ingénieur, et surtout l'ingénieur des mines, ressemble au chef d'orchestre dont la maîtrise musicale, même sans être vertueuse, permet de jouer un rôle de coordination indispensable à la réalisation d'une symphonie ${ }^{31}$. Dans cette mesure, l'ingénieur est l'acteur principal de la mise en place de l'OST et il concourt à la circulation de ses techniques. La conclusion de l'article de P. Audibert confirme cette attitude par la reprise de la préface d'Henri Le Châtelier dans l'ouvrage de J. Amar :

«Il est inutile de mettre en trop grande lumière une cuisine préparatoire dont les détails, parfois difficiles à comprendre, peuvent donner lieu à des malentendus; il faut seulement montrer aux ouvriers le résultat final, l'augmentation de salaires que les méthodes scientifiques leur permettent de réaliser à coup sûr " $^{32}$.

Dans le cas de Pertusola, l'« esprit de l'ingénieur » se traduit en expériences que l'on peut retrouver décrites dans les rapports techniques. Par exemple, dans certaines expériences effectuées sur le herschage à la main, deux ouvriers doivent pousser un wagonnet équipé d'un dynamomètre, placé sur un circuit de rails d'une cinquantaine de mètres, jusqu'à l'épuisement des forces. La "base des données " ainsi obtenue est alors transposée sur un plan cartésien. Dans le commentaire final, l'ingénieur propose une comparaison analogique avec les ergogrammes de Mosso ${ }^{33}$.

Malgré l'apparence cartésienne de la méthode utilisée, on ne peut pas éviter de remarquer la dureté du traitement réservé aux ouvriers-testeurs. L'attention des ingénieurs est consacrée aux seules conditions pouvant impacter sur la justesse des enregistrements, voire, par exemple, si l'ouvrier s'arrête pour épuisement ou pour insouciance. Dans ce dernier cas, l'effort d'évaluation retombe dans une catégorisation arbitraire et moralisante. Le travailleur représenté par les courbes Gennaper apparait comme une entité physico-mécanique, mesurée à travers l'application des lois de Coulomb et Euler (modifiées). Les observations psycho-physiologiques semblent avoir été oubliées ou réduites à un complément pour la détermination de l'ouvrier-type.

Pourtant, la technique Gennaper fait partie d'un plus vaste projet d'organisation scientifique qui réabsorbe les variables psycho-physiologiques par d'autres voies. La simplification de l'évaluation est contrebalancée en fait par une action techno-politique 
de régulation du milieu ouvrier. Par exemple, la propagande hygiéniste de la compagnie met l'accent sur l'importance de la propreté en fonction de la récupération énergétique des travailleurs. Ce discours, qu'on appellerait plus traditionnellement paternaliste, obtient dans ce contexte une nuance "rationalisante", car il est mis au profit de la gestion de la performance ouvrière. Cette stratégie fait écho à certaines positions du BIT (Bureau Internationale du Travail) - dont les actes des Congrès des années 1927 et 1929 apparaissent dans l'article d'Audibert et, parfois, dans les archives. À cette époque, en effet, on indique l'organisateur (que nous appellerions aujourd'hui le "manager») comme l'acteur qui devrait prendre en charge les formes symptomatiques de la fatigue usure, surmenage épuisement - par l'exercice de l'orientation professionnelle et de l'amélioration des conditions du travail ${ }^{34}$. Comme on voit dans le cas de Pertusola, cette prise en charge déborde facilement vers un modèle de contrôle intégral du corps du travail.

La conception de la fatigue chez les ingénieurs de Pertusola est donc plus complexe que ce que l'on pourrait penser en analysant seulement les expériences des rapports techniques. En tout cas, l'élaboration de la nouvelle méthode d'évaluation se fait directement sur le carreau de la mine, par l'observation expérimentale des hommes en mouvement. La mine devient alors une sorte de laboratoire où on isole et on étudie les tâches en situation " contrôlée ", mais où on réalise aussi que cette modélisation ne peut apporter qu'une solution partielle au problème de la fatigue.

\section{Conclusion}

Avec la publication dans la Revue de l'Industrie Minérale, la méthode Gennaper obtient un baptême scientifique qui sera confirmé, en 1937, par la remise du Prix Fayol à P. Audibert. Le Gennaper est utilisé chez Pertusola jusqu'aux années 1950, et, malgré son caractère singulier, il représente un cas d'étude utile pour observer l'univers des connaissances sous-jacentes à une approche technique de la question du travail. Dans des moments de crise et de transformation comme l'entre-deux-guerres, les ingénieurs élaborent des solutions managériales reposant sur des références variées, mais réduites de par leur faisabilité technique. Comme cela a été souvent précisé, la rationalisation apparaît donc finalement comme un dispositif de discipline au travail, soutenu par un certain esprit culturel scientiste qui trouve un terrain d'accueil favorable dans le corps des ingénieurs de l'entre-deux-guerres.

Dans l'imaginaire des organisateurs, l'action de rationalisation dépend alors d'une vision organique dont la visée finale est de standardiser et fluidifier le cycle productif pour en augmenter la productivité. L'intervention régulatrice - à caractère " techno-politique $\|^{35}$ - porte alors sur les acteurs de la production et prend la forme d'une discipline quantitative (l'intensification de l'allure) et/ou qualitative (l'optimisation du mouvement). L'objectif est d'établir des barèmes d'évaluation applicables aux travailleurs et non pas de donner une définition de la fatigue en tant que telle. En ciblant les «temps morts», qui donnent la mesure du "geste inutile", la fatigue est perçue comme une dysfonction mécanique et la dimension physiologique - où la fatigue se présente comme « intoxication ", pour reprendre le terme du physiologiste Angelo $\mathrm{Mosso}^{36}$ - est alors récupérée dans le contexte plus stratégique du projet d'OST. Le cas de mines de Pertusola montre ainsi de manière très vive les effets de la combinaison d'un paternalisme disciplinaire avec les 
principes d'une rationalisation qui prétend légitimer "scientifiquement» des choix reposant plutôt sur une évaluation que sur un jugement moral des hommes au travail.

Dans ce contexte, la stratification de la rationalisation et de la crise minière de l'entredeux-guerres contribue à définir une vision du monde du travail où la figure de l'ingénieur est hégémonique. Aussi, dans une certaine mesure, les ingénieurs peuvent être décrits comme "passeurs " - ou intermédiaires - des connaissances scientifiques, techniques et managériales dans une dynamique de circulation adaptative ${ }^{37}$. L'histoire du Gennaper souligne justement l'existence de cette circulation des savoirs, non seulement transnationale et interconnectée, mais aussi multi-scalaire, impliquant les techniciens sur le terrain et la communauté scientifique supranationale. La mise à l'épreuve de ces théories met aussi en évidence que cette circulation des connaissances scientifiques a des retombées importantes sur la vie ouvrière.

Les travailleurs, pour leur part, opposent une certaine résistance à ces outils techniques de manière à la fois ouverte, violente et épisodique, mais aussi indirecte et quotidienne, mêlant insubordination, freinage, absentéisme, turnover et permanence d'anciennes habitudes du travail. Comme on l'a vu dans la première partie de l'article, la résistance et le conflit jouent un rôle non négligeable dans le processus de création des techniques, qui est modifié et adapté afin de contourner, réprimer ou trouver un accord avec les opposants. L'adaptabilité de la technique peut donc être balayée comme une sorte d'outil de négociation. Notre cas d'étude semble alors suggérer une piste d'analyse concernant la transformation des savoirs scientifiques sur la fatigue humaine au $\mathrm{XX}^{\mathrm{e}}$ siècle. Dans ce cadre, l'observation des usages économiques et industriels de ces connaissances pourrait donner de nouvelles perspectives à l'interprétation de l'organisation scientifique du travail et de la construction du management des ressources humaines.

\section{NOTES}

1. Pour un panorama de la littérature scientifique sur l'étude du travail humain, on renvoie à VATIN François, Le travail et ses valeurs, Paris, Albin Michel, 2008.

2. Voir, par exemple DURRIVE Barthélemy « Deux ouvriers-machine, avant et après Taylor ", L'Homme et la société, n² 205, mars 2017, p.53-86.

3. Cette expression est utilisée dans le sens qui lui est donné par Giulio Sapelli dans son ouvrage SAPELLI, Giulio, Gli « organizzatori della produzione » tra struttura d'impresa e modelli culturali, Milano, Einaudi, 1981. Dans cet article, nous considérons les professionnels techniciens de l'industrie comme les acteurs clé de la rationalisation. Parmi eux, les ingénieurs ayant un rôle de management ou de direction ont une fonction centrale dans notre analyse.

4. NELSON Daniel, A Mental Revolution. Scientific Management after Taylor, Columbus, Ohio State University Press, 1992, p.14.

5. Il s'agit d'une approche assez pratiquée par les consultants et adaptée plus ou moins directement de l'expérience taylorienne. Au sujet des approches de la consultance, voir KIPPING Matthias, WRIGHT Christopher, «Consultants In Context : Global Dominance, 
Societal Effect and the Capitalist System ", dans CLARK Thimothy, KIPPING Matthias, The Oxford Handbook of Management Consulting, Oxford, Oxford University Press, 2012, p.165-185.

6. Pour les détails techniques du système Bedaux, voir LALOUX Philippe, Le système Bedaux de calcul des salaires, Paris, Éditions Hommes \& Techniques, 1951. Pour une analyse historique sur la consultance Bedaux, voir WRIGHT Christopher, KIPPING Matthias « The Engineering Origins of the Consulting Industry and their Long Shadow ", dans CLARK Thimothy, KIPPING Matthias (ed.), The Oxford Handbook of Management Consulting, Oxford, Oxford University Press, 2012, p.165-185.

7. La référence principale pour l'histoire de l'application du système Bedaux aux mines Pertusola est ROLLANDI Maria Stella, « Il sistema Bedaux nelle miniere sarde della « Pertusola (1927-1935) », Studi storici: rivista trimestrale dell'Istituto Gramsci, vol.26, n ${ }^{\circ}$, 1985, p.69-106.

8. « Rapporto dei capi servizio, giugno-novembre 1929 », Fondo Crotone, San giovanni 1928-1929, Rapporti mensili miniere Pertusola, ASM ; « Secondo Rapporto Tecnico Generale », 30 novembre 1930, Fondo Pertusola, Rapporti Tecnico Generali, ASMM.

9. Pour une analyse de la condition socio-économique de la Sardaigne de l'époque, voir CORSI Angelo, L'azione socialista tra i minatori della Sardegna,1898-1922 : contributo allo studio del movimento operaio italiano, Edizioni di Comunità Luogo, Milano, 1959; ROLLANDI Maria Stella, Miniere e minatori in Sardegna, dalla crisi del primo dopoguerra alla nascita di Carbonia (1919-1939), Cagliari,1981.

10. ROLLANDI Maria Stella, «Il sistema Bedaux... » op. cit.

11. Pour la position des syndicats fascistes et de T. Cianetti au sujet de l'OST, voir FASSIO, Mario « Bedaux o Taylor ? ", L'informazione industriale, 17/09/1930 cit. in SAPELLI, Giulio, « Gli organizzatori...», op. cit., 1981, p. 651-652 ; MUSSO Stefano, La gestione della forza lavoro sotto il fascismo. Realizzazione e contrattazione collettiva nell'industria metallurgica torinese (1910-1940), Franco Angeli, 1987 ; SAPELLI, Giulio, « Appunti per una storia dell'organizzazione scientifica del lavoro in Italia », in Quaderni di sociologia, 1976, n²-3, p.151-171.

12. «Terzo Rapporto Tecnico Generale », 30 giugno 1931, Fondo Pertusola, Rapporti Tecnico Generali, ASMM.

13. ROLLANDI Maria Stella, « Il sistema Bedaux... » op. cit., 1981, p.90.

14. PERUGINI Mario, Il farsi di una grande impresa. La Montecatini fra le due guerre mondiali, Milano, Franco Angeli, 2014, p.101.

15. BIGAZZI Duccio, « Gli operai della catena di montaggio : la FIAT 1922-1943 », Annali Feltrinelli, XXI, 1979-1980, Milano, 1981, p.926-927.

16. Dans d'autres cas, on a également essayé de contourner l'opposition ouvrière à travers des escamotages lexicaux : voir, par exemple, le cas de la Lukens Steel Company décrit dans KIPPING Matthias, «Consultancy and Conflicts : Bedaux at Lukens Steel and the Anglo-Iranian Oil Company », Entreprises et histoire, vol.2, n²5, 2000, p.9-25. Cette possibilité fut évoquée et, dans une certaine mesure, mise en place chez Pertusola : voir ROLLANDI Maria Stella, op. cit.

17. AUDIBERT Paul, «Généralités sur l'organisation rationnelle dans les mines », Revue de l'Industrie Minérale, vol. 26, n453, juillet 1933, p.355-385; Id. « Le système Gennaper pour l'évaluation des tâches ", Revue de l'Industrie Minérale, n³54, 1935, p.429-454; Id.

"Compléments à l'exposition du système Gennaper pour l'évaluation des tâches ", Revue de l'Industrie Minérale, $\mathrm{n}^{\circ}$ 405, 1937, p.565-570.

18. AUDIBERT Paul, « Généralités ... », op.cit., 1933. 
19. Ibidem.

20. Ibid, p.371.

21.

On peut noter d'ailleurs que l'auteur s'inspire de l'ouvrage de Jules Amar pour adopter cette classification.

22. « Rapporto Tecnico del Mercolédi », 5 avril 1933, Fondo Pertusola, Rapporto Tecnici del Mercoledi San Giovanni, ASMM.

23. Sur l'analogie dans le développement du savoir technique, voir BEAUNE Sophie de, HILAIRE-PÉREZ Liliane, VERMEIR Koen (dir.), L'analogie dans les techniques, Paris, CNRS, 2017.

24. Sur le geste technique, consulter GARÇON Anne-Françoise, « Des modes d'existence du geste technique », e-Phaïstos, IV-2, 2015, p.84-92. L'interprétation de la fatigue comme surmenage causé par une mauvaise organisation technique est déjà perceptible dans l'œuvre de Taylor. Voir WALLON Henri, « Taylorisme, rationalisation, sélection, orientation ", Perspectives interdisciplinaires sur le travail et la santé, 14, n², 2012, consulté le 02 mars 2018, URL : http://journals.openedition.org/pistes/2612.

25. SAND René, Organisation industrielle, médecine sociale et éducation civique en Angleterre et aux États-Unis, Paris, Baillière, 1920.

26. AMAR Jules, Le moteur humain et les bases scientifiques du travail professionnel,

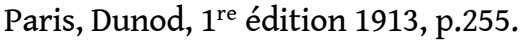

27. «Quinto Rapporto Tecnico Generale », 27 novembre 1933, Fondo Pertusola, Rapporti Tecnico Generali, ASMM.

28. « Abrégé de la méthode Gennaper », Fondo Pertusola, Caratteristiche Bedaux, ASMM.

29. Comme il a été en revanche proposé par la littérature sur le sujet. Voir ROLLANDI Maria Stella, op.cit. ; ZANDA Romeo, Mutamenti dei sistemi retributivi nelle miniere sarde. Da Taylor a Bedaux, Cagliari, Giuffré, 2003.

30. Pour la question de la formation des ingénieurs voir GRELON André (dir.) Les Ingénieurs de la crise : titre et profession entre les deux guerres, Paris, Éditions de l'EHESS, 1986 ; BRIANTA Donata, Europa Mineraria, circolazione delle élites e trasferimento tecnologico (secoli XVIII ${ }^{\mathrm{e}}$-XIX) ), Franco Angeli, 2007 ; PASSAQUI JeanPhilippe, Les voyages forment l'ingénieur, les houillères du Centre et du Midi de la France, 1851-1873, Paris, Cl. Garnier, 2015.

31. JAMET Philippe, « Préface. Civil par dessus tout... », in BERTILORENZI Marco, PASSAQUI Jean-Philippe, GARÇON Anne-Françoise (dir.), Entre technique et gestion. Une histoire des « ingénieurs civils des mines » (XIX ${ }^{e}-X^{e}$ siècles), Paris, Presses des Mines, 2016. On peut y voir non seulement les traces d'une culture d'ingénieur éminemment française, mais aussi l'influence de la vision de la rationalisation qui caractérise le contexte italien. Voir RICCIARDI Ferruccio, « Une utopie conservatrice. Rationalisation et sciences du travail dans l'Italie fasciste ", Vingtième Siècle. Revue d'histoire, n¹36, 2017.

32. AUDIBERT Paul, « Généralités ... », op. cit., 1933, p.384.

33. « Rapporto Tecnico del Mercoledi $n^{\circ} 3$ », 1934, Fondo Pertusola, Rapporti Tecnici del Mercoledi Ingurtosu, ASMM.

34. Voir notamment les travaux de Isabelle Lespinet-Moret au sujet de l'OIT et du BIT. 35. Le terme « techno-politique » est inspiré par HECHT Gabrielle, Le Rayonnement de la France. Énergie nucléaire et identité nationale après la Seconde Guerre mondiale, Paris, Éditions Amsterdam, 2014. Toutefois, la composante politique s'exprime ici au niveau d'une échelle plus réduite, liée à l'organisation des sites industriels et des communautés 
ouvrières qui en dépendent. On peut également consulter : HECHT Gabrielle, Uranium africain, une histoire globale, Paris, Éditions Amsterdam, 2014.

36. MOSSO Angelo, La fatica, Milano, Treves, 1891. Jules Amar reprend cette définition dans AMAR Jules, Le moteur humain..., op.cit., 1913, p.301.

37. On peut se référer ici à la littérature sur la figure professionnelle de l'ingénieur dont par exemple THEPOT, André (dir.), L'Ingénieur dans la société française, Paris, Les Éditions ouvrières, 1985. Pour la question de la circulation, voir, entre autres, HILAIRE PEREZ Liliane, ZAKHAROVA Larissa (dir.), Les techniques et la globalisation au XX $X^{e}$ siècle, Rennes, PUR, 2016. Pour la circulation dans le monde minier, voir BRIANTA Donata, Europa Mineraria..., op.cit., 2007.

\section{RÉSUMÉS}

Le problème de l'efficience à la mine est, à la base, un problème de travail humain. Dans le contexte de la rationalisation d'entre-deux-guerres, on voit la naissance de plusieurs modèles d'évaluation qui proposent d'optimiser la performance du corps au travail par la gestion de sa dépense énergétique. C'est le cas, par exemple, de la méthode Gennaper, développée au sein de la Compagnie Minière Pertusola (branche italienne du groupe franco-espagnol Peñarroya) à partir d'une réflexion sur la nature du travail humain. Sa conception croise plusieurs chemins de connaissance, en premier lieu les études sur la fatigue humaine des XVIII ${ }^{\mathrm{e}}$ et $\mathrm{XIX}^{\mathrm{e}}$ siècles et les applications de la science de l'organisation rationnelle. Elle relève aussi d'une circulation transnationale des savoirs techniques activée par les ingénieurs. Cette contribution vise donc à définir la manière dont le savoir sur la fatigue humaine est mis au profit d'une expérience de rationalisation à la mine.

Efficiency in mining industry is clearly a matter of human labour. During the interwar period the efficiency problem was frequently approached in terms of evaluation of individual perfomances. This was the general leitmotiv of industrial consultants who provided several models of evaluation to cope with it. This contribution aims to analyse one of these methods, called Gennaper. Gennaper was developed by some mining engineers of Pertusola Mining Company, owned by Peñarroya group, as a result of a scientific investigation on human labour. Although their purpose was really practical, they needed some theoretical bases for the study and they found them in the literature of various disciplines, from medicine to physics. So it seems interesting to retrace the different phases of the study in order to observe what were the contents in which engineers were interested and how they used them. This case will also provide an exemple of scientific management in practice, which reveals a circular exchange of knowledge and techniques about human labour. 
INDEX

Mots-clés : histoire des techniques, entre-deux-guerres, travail, mine, Peñarroya.

Index chronologique : Époque contemporaine

Index géographique : Europe

Keywords : history of technology, interwar period, scientific management, mining industry, Peñarroya, human labour

\section{AUTEUR}

\section{FRANCESCA SANNA}

Francesca Sanna est doctorante contractuelle en Histoire à l'Université Paris Diderot Paris 7 et rattachée au laboratoire interdisciplinaire LIED (Laboratoire Interdisciplinaire pour les Énergies de Demain). Son projet de thèse s'intitule «Mesurer l'effort, rémunérer la fatigue : le système Bedaux et la gestion de la souffrance ouvrière dans l'industrie minière européenne (1930-1960) », sous la direction de Mathieu Arnoux (U. Paris 7) et Manuela Martini (U. Lyon 2). Pendant son doctorat, elle a collaboré avec l'équipe EQUIPEX DFIH sur un projet de base de données financières historiques de la Bourse de Paris. 